\title{
Separation of the 5- and 6-Carboxy Regioisomers of ROX and JOE Dyes with Examples of $\mathrm{N}$-(3-Azidopropyl)amide Synthesis
}

\author{
Nadezhda S. Baleeva ${ }^{a}$ \\ Marina B. Zagudaylova \\ Mikhail S. Baranov*a,b (D) \\ a Institute of Bioorganic Chemistry, Russian Academy of Scienc- \\ es, Miklukho-Maklaya 16/10, 117997 Moscow, Russia \\ baranovmikes@gmail.com \\ b Pirogov Russian National Research Medical University, Ostrovi- \\ tianov 1, 117997, Moscow, Russia
}
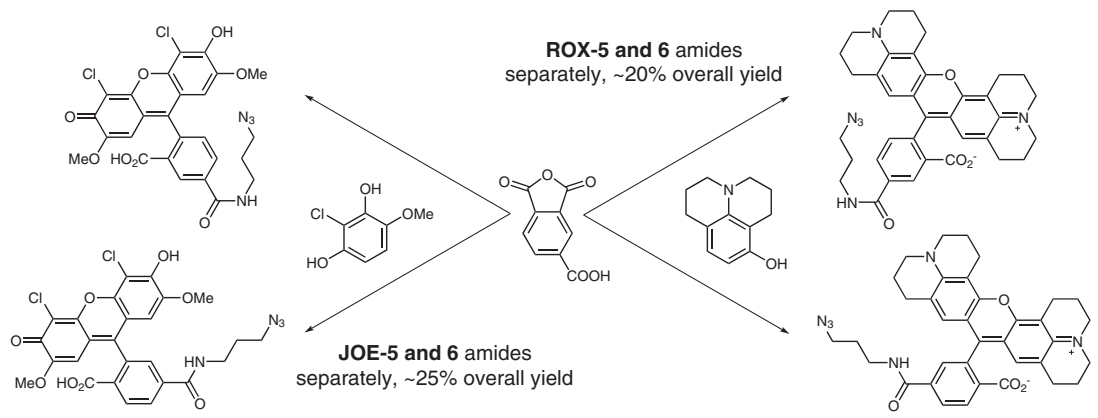

Received: 23.05.2018

Accepted after revision: 01.07.2018

Published online: 25.07 .2018

DOI: 10.1055/s-0037-1610216; Art ID: so-2018-d0034-psp

License terms: (c)

Abstract Despite the widespread applications of various rhodamine and fluorescein 5- and 6-carboxy derivatives, the preparation of their pure regioisomers, in particular cases, remains a complex task. In the present paper we propose optimized approaches to the synthesis and separation of these isomers of ROX and JOE dyes, and also demonstrate their applicability in the synthesis of the corresponding $\mathrm{N}$-(3-azidopropyl)amides.

Key words fluorescent dyes, fluorescein, rhodamine, JOE, ROX, clickchemistry, azide

Various rhodamine and fluorescein derivatives, as well as other xanthene dyes, have long attracted the interest of researchers, and such compounds are actively used for fluorescent labeling in biological research. ${ }^{1}$ Their 5 - and 6 -carboxy derivatives are typically used for binding with target objects or for other functionalization (Figure 1).
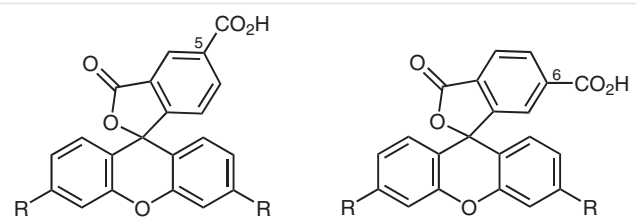

$\mathrm{R}=\mathrm{OH}, \mathrm{NR}_{2}^{\prime}$

Figure 1 The 5-and 6-carboxy derivatives of xanthene dyes

Despite the widespread applications of these carboxy derivatives, it is surprisingly difficult to obtain these compounds as pure regioisomers. The separation of these isomers is well enough developed for unsubstituted fluoresce- in $^{2}$ and several rhodamines, ${ }^{3}$ but for other derivatives, quite complex procedures are used. In particular, such separation (as indeed the synthesis itself) is difficult for one of the most redshifted amino derivatives - ROX (rhodamine 101 or X-rhodamine), as well as for a novel $4^{\prime}, 5^{\prime}$-dichloro-2',7'dimethoxyfluorescein - JOE, which has recently been actively used as an analogue of an even more inaccessible hexachlorofluorescein (HEX). ${ }^{4}$ The most typical separation methods for obtaining of 5- and 6-carboxy-X-rhodamines are the use of difficult to obtain phthalic mono-aldehydes ${ }^{5}$ or chromatographic separation of the final products on large columns using gradient eluting systems, ${ }^{5 a, 6}$ which lead to large losses due to oxidation processes. Typically, isomers of various halogenated carboxyfluoresceins can be separated via fractional crystallization of their lactone diester diethylammonium salts, ${ }^{4 a}, 7$ which is accompanied by a noticeable material loss (at least for one of the isomers), while an efficient yielding column separation of JOE isomers has been reported only for one example of the pentafluorophenyl esters. ${ }^{4 b}$

In the present paper we propose optimized approaches to the synthesis and separation of the 5- and 6-carboxy isomers of ROX and JOE dyes, and also demonstrate their applicability in the synthesis of the corresponding $N$-(3-azidopropyl)amides, which are widely used for fluorescent labeling of alkyne-modified oligonucleotides and other targets, which are applied in various fields of biology. ${ }^{4 c, 8}$

Surprisingly while these derivatives are widely commercially available, their synthetic pathways are still not described in the scientific literature, except for one of the ROX isomers. ${ }^{9}$

The key finding that enabled us to improve the yield significantly and readily separate the 5- and 6-carboxy-Xrhodamines was a stepwise synthesis (Scheme 1 ). 


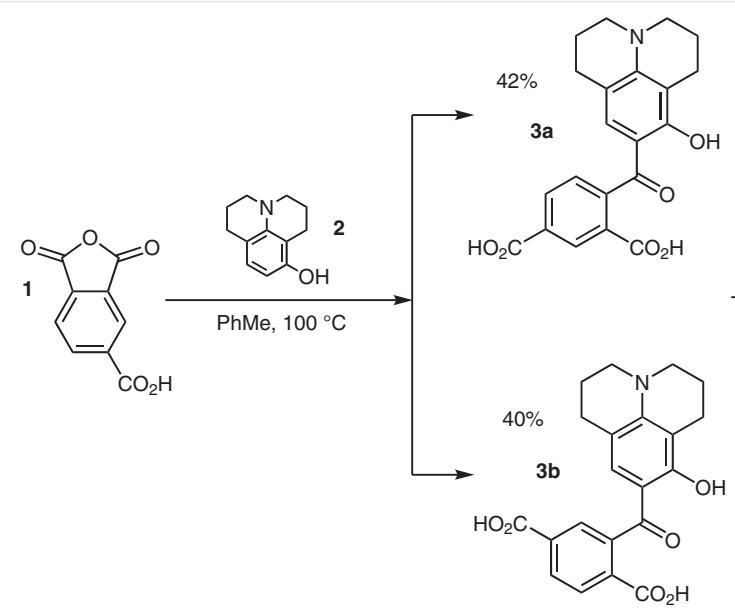

overall yield $>80 \%$

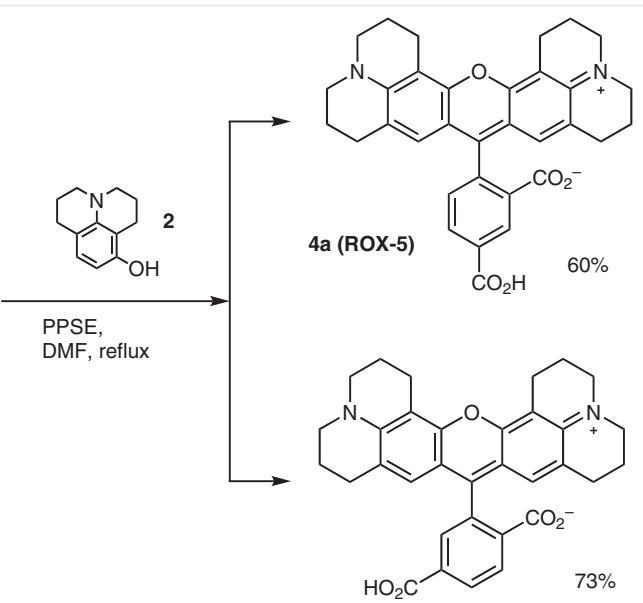

$4 b(R O X-6)$

Scheme 1 Preparation of 5- and 6-carboxy-X-rhodamines 4

Usually, the condensation of aminophenol $\mathbf{2}^{10}$ with 4carboxyphthalic anhydride $\mathbf{1}$ is carried out under the action of strong acids and heating (for example, by heating to reflux in propanoic or butanoic acid in the presence of $\mathrm{H}_{2} \mathrm{SO}_{4}$ or PPSA ${ }^{5,6}$ ), leading directly to the formation of xanthene products. However, the intermediate ketones $\mathbf{3}$ can be obtained in the absence of strong acids and with a different ratio of the starting materials. It is known from the literature that the isomers have quite different properties, which has previously allowed their separation by recrystallization in the case of dimethylaminophenol, ${ }^{3}$ allowing isomer $\mathbf{3 a}$ to be isolated after condensation of compounds $\mathbf{1}$ and $\mathbf{2}$ in acetic acid. ${ }^{11}$ Replacing the solvent with toluene allowed us to synthesize in good yield both compounds $\mathbf{3 a}$ and $\mathbf{3 b}$, which possessed markedly different properties: substance $\mathbf{3 b}$ was amorphous and readily soluble in diethyl ether, whereas isomer 3a was crystalline and practically insoluble. In this regard, after the polymeric by-products were removed using short flash chromatography, the majority of compound 3a was isolated from the mixture by simple trituration with ether. Moreover, the remaining mixture of substances 3a and $\mathbf{3 b}$ was separated by straightforward chromatography on silica gel, eluting with $\mathrm{CHCl}_{3} / \mathrm{MeOH} / \mathrm{Et}_{3} \mathrm{~N}$.

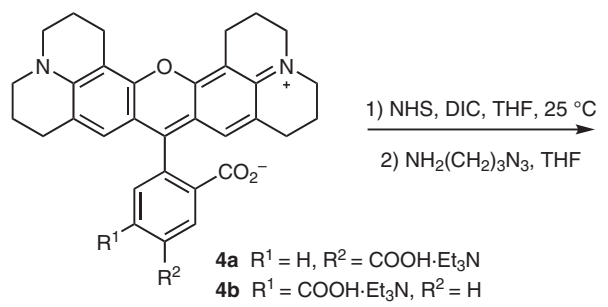

The subsequent condensation to give the corresponding carboxy-X-rhodamines was carried out with an additional equivalent of aminophenol $\mathbf{2}$ in $\mathrm{N}, \mathrm{N}$-dimethylformamide (DMF) using trimethylsilyl polyphosphate as a dehydrating agent, itself readily obtained from hexamethyldisiloxane and $\mathrm{P}_{2} \mathrm{O}_{5}$. The resulting isomers of carboxy-X-rhodamines were obtained in good purity (more than $80 \%$ by NMR analysis); however, flash chromatography $\left(\mathrm{CHCl}_{3} / \mathrm{MeOH} / \mathrm{Et}_{3} \mathrm{~N}\right)$ allowed them to be obtained in a purer form and to be converted into the stable triethylammonium salts.

The final conversion of these derivatives into $\mathrm{N}$-(3-azidopropyl)-amides $\mathbf{5}$ is also most efficiently carried out in a stepwise manner, with isolation and crude purification of the intermediate $N$-hydroxysuccinimide ester, which makes it possible to obtain product $\mathbf{5}$, not only in good yield, but also in high purity (Scheme 2).

The synthesis of 5- and 6-carboxy derivatives of JOE started from 4-methoxyresorcinol 8, which is not commercially available but can be synthesized from isovanillin 6 . This synthesis was optimized by Tsybulsky et al. ${ }^{4 b}$ but it may be noted that gaseous chlorine used in the first step can be conveniently replaced with sulfuryl chloride (Scheme 3).

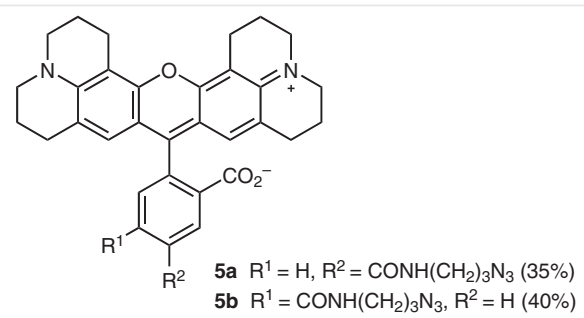

Scheme 2 Synthesis of 3-azidopropylaminocarbonyl-X-rhodamines 5 
<smiles>COc1ccc(C=O)cc1O</smiles><smiles>COc1ccc(C=O)c(O)c1Cl</smiles>

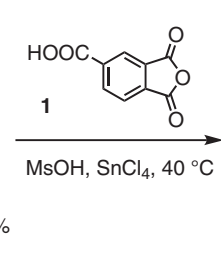

9a (JOE-5) $R^{1}=\mathrm{H}, \mathrm{R}^{2}=\mathrm{COOH}$ $9 b\left(\right.$ JOE-6) $\mathrm{R}^{1}=\mathrm{COOH}, \mathrm{R}^{2}=\mathrm{H}$

Scheme 3 Synthesis of 5- and 6-carboxy-JOE dyes 9

Several methods for condensing the resulting resorcinol $\mathbf{8}$ with 4-carboxyphthalic anhydride $\mathbf{1}$ are presented in the literature. ${ }^{4}$ However, we confirmed that simply gentle heating in the presence of tin chloride, as proposed by Tsybulsky et al. ${ }^{\mathrm{b}}$ reproducibly leads to the formation of compound 9. It should be noted that the isomers can be separated even at this stage using a chromatographic system of $\mathrm{NH}_{3}$ in EtOH on silica $\left(R_{f}=0.30\right.$ and 0.45 in $\mathrm{EtOH} / \mathrm{NH}_{3}$ (sat, aq), 9:1).

However, even more convenient is the sequence previously proposed for fluorescein ${ }^{2 b}$ associated with the use of acetylated $\mathrm{N}$-succinimidyl esters 11 (Scheme 4), which are well-separated chromatographically on silica using toluene/EtOAc mixtures.
The esters obtained this way can be readily converted into amides by the action of various primary amines, leading directly to the desired amides of JOE; distinguishing this method from the approach using a cyclohexanecarbonyl protective group, ${ }^{4 \mathrm{~b}}$ removal of which requires additional effort.

Of course, such an approach leads to the loss of two equivalents of the amine used, but this is not critical against the background of the total cost of the whole synthesis. As an example, this transformation was carried out by us to furnish the corresponding $\mathrm{N}$-(3-azidopropyl)-amides $\mathbf{1 2}$, which were obtained with yields of more than $75 \%$ each (Scheme 5).

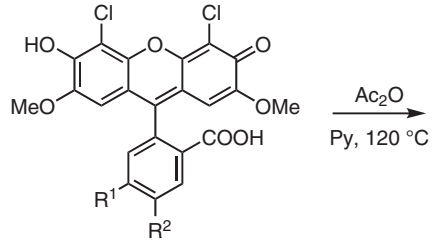

9a $\mathrm{R}^{1}=\mathrm{H}, \mathrm{R}^{2}=\mathrm{COOH}$ 9b $\mathrm{R}^{1}=\mathrm{COOH}, \mathrm{R}^{2}=\mathrm{H}$<smiles>[R]c1cc2c(cc1[2H])C1(OC2=O)c2cc(OC)c(OC)c(OC)c2Oc2c(Cl)c(OC)c(OC)c(Cl)c21</smiles>

10a $\mathrm{R}^{1}=\mathrm{H}, \mathrm{R}^{2}=\mathrm{COOH}$ 10b $\mathrm{R}^{1}=\mathrm{COOH}, \mathrm{R}^{2}=\mathrm{H}$

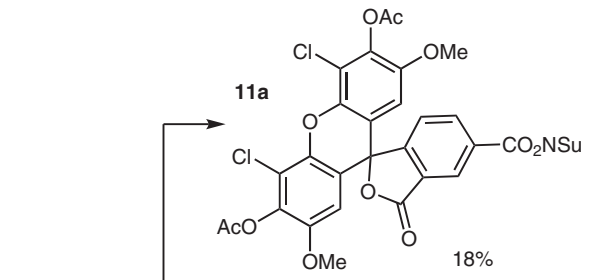

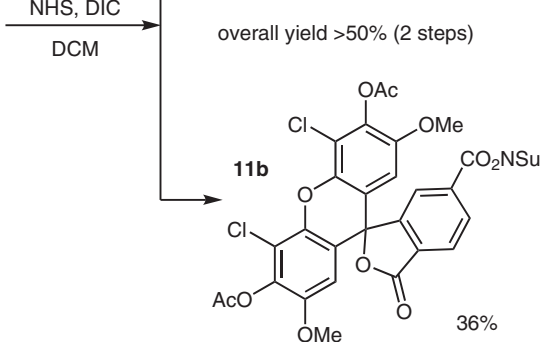

Scheme 4 Synthesis and separation of 5- and 6-carboxy-4',5'-dichloro-2',7'-dimethoxyfluorescein derivatives 11
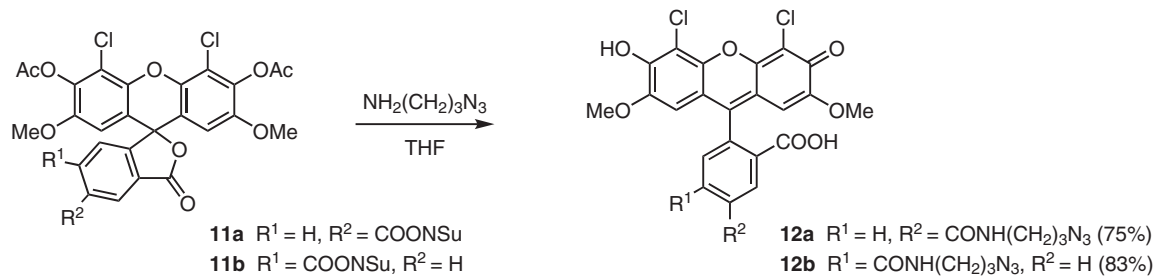

Scheme 5 Synthesis of 3-azidopropyl-jOE derivatives 12 
Commercially available reagents were used without additional purification. E. Merck Kieselgel 60 was used for column chromatography. Thin-layer chromatography (TLC) was performed on silica gel 60 F254 glass-backed plates (MERCK). Visualization was effected by UV light (254 or $312 \mathrm{~nm}$ ) and staining with $\mathrm{KMnO}_{4}$.

NMR spectra were recorded with a $700 \mathrm{MHz}$ Bruker Avance III NMR at $293 \mathrm{~K}$, a $800 \mathrm{MHz}$ Bruker Avance III NMR at $333 \mathrm{~K}$, and a Bruker Fourier 300. Chemical shifts are reported relative to residual peaks of $\mathrm{CDCl}_{3}$ $\left(\delta=7.27 \mathrm{ppm}\right.$ for ${ }^{1} \mathrm{H}$ and $\delta=77.0 \mathrm{ppm}$ for $\left.{ }^{13} \mathrm{C}\right), \mathrm{D}_{2} \mathrm{O}(\delta=4.79 \mathrm{ppm}$ for $\left.{ }^{1} \mathrm{H}\right)$ or DMSO- $d_{6}\left(\delta=2.51 \mathrm{ppm}\right.$ for ${ }^{1} \mathrm{H}$ and $\delta=39.5 \mathrm{ppm}$ for $\left.{ }^{13} \mathrm{C}\right)$. Melting points were measured with an SMP 30 apparatus. High-resolution mass spectra (HRMS) spectra were recorded with an LTQ Orbitrap XL (ThermoFisher Scientific, USA) equipped with a dual-nebulizer ESI source.

\section{Procedures}

\section{Trimethylsilyl Polyphosphate (PPSE) in Solution}

A mixture of phosphorus pentoxide ( $20 \mathrm{~g}, 70 \mathrm{mmol})$, hexamethyldisiloxane $(50 \mathrm{~mL}, 247 \mathrm{mmol})$ and chloroform $(100 \mathrm{~mL})$ was heated at reflux for $1 \mathrm{~h}$ until the solution was clear, then the mixture allowed to cool to r.t. The reagent solution may be stored at $4{ }^{\circ} \mathrm{C}$ for 3 months.

\section{(8-Hydroxyjulolidine-9-carbonyl)phthalic Acids 3}

To a stirred solution of 8-hydroxyjulolidine $\mathbf{2}(1.89 \mathrm{~g}, 10 \mathrm{mmol})$ in toluene (30 mL), 4-carboxyphthalic anhydride $1(2.3 \mathrm{~g}, 12 \mathrm{mmol})$ was added at $60^{\circ} \mathrm{C}$ and the reaction mixture was stirred for $48 \mathrm{~h}$ at $100^{\circ} \mathrm{C}$. After cooling to r.t., the solvent was decanted and the residue was dissolved in $\mathrm{CHCl}_{3} / \mathrm{MeOH}$ (60:40) and filtered through a thin layer of sili$\mathrm{ca}$, collecting slightly colored fractions. The resulting mixture of isomers $3 \mathbf{a}$ and $\mathbf{3 b}$ was heated to reflux in $\mathrm{Et}_{2} \mathrm{O}(75 \mathrm{~mL})$, cooled to r.t., filtered, and washed with $\mathrm{Et}_{2} \mathrm{O}(25 \mathrm{~mL})$ to give pure $3 \mathbf{a}$ as a yellow crystalline solid $(1.1 \mathrm{~g})$. The mother liquor was evaporated and the residual mixture of $\mathbf{3 a}$ and $\mathbf{3 b}$ was separated by column chromatography $\left(\mathrm{CHCl}_{3} / \mathrm{MeOH} / \mathrm{Et}_{3} \mathrm{NEt}_{3} \mathrm{~N}, 75: 20: 5\right)$ to afford compounds $\mathbf{3 a}$ and $\mathbf{3 b}$ as their triethylammonium salts. The obtained salts were dissolved in EtOAc $(300 \mathrm{~mL})$ and washed with $\mathrm{HCl}(3 \%, 5 \times 100 \mathrm{~mL})$. The aqueous phase was extracted with EtOAc $(3 \times 50 \mathrm{~mL})$ and the combined organic layers were washed with brine, dried over $\mathrm{Na}_{2} \mathrm{SO}_{4}$, filtered, and evaporated.

\section{4-(8-Hydroxyjulolidine-9-carbonyl)isophthalic Acid (3a)}

Overall yield: $1.6 \mathrm{~g}$ (42\%); yellow solid; m.p. $>210{ }^{\circ} \mathrm{C}$ (decomp.); $R_{f}=$ $0.44\left(\mathrm{CHCl}_{3} / \mathrm{MeOH} / \mathrm{Et}_{3} \mathrm{~N}, 70: 25: 5\right)$.

${ }^{1} \mathrm{H}$ NMR (700 MHz, DMSO- $d_{6}$ ): $\delta=13.30$ (br. s., $2 \mathrm{H}, \mathrm{COOH}$ ), 12.83 (s, $1 \mathrm{H}, \mathrm{OH}), 8.11(\mathrm{dd}, J=8.1,1.3 \mathrm{~Hz}, 1 \mathrm{H}, \mathrm{Ar}), 8.03(\mathrm{~d}, J=8.1 \mathrm{~Hz}, 1 \mathrm{H}, \mathrm{Ar})$, $7.78(\mathrm{~d}, J=1.3 \mathrm{~Hz}, 1 \mathrm{H}, \mathrm{Ar}), 6.41(\mathrm{~s}, 1 \mathrm{H}, \mathrm{Ar}), 3.23-3.27\left(\mathrm{~m}, 4 \mathrm{H}, \mathrm{CH}_{2}\right)$, $2.59\left(\mathrm{t}, J=6.4 \mathrm{~Hz}, 2 \mathrm{H}, \mathrm{CH}_{2}\right), 2.42\left(\mathrm{t}, J=6.0 \mathrm{~Hz}, 2 \mathrm{H}, \mathrm{CH}_{2}\right), 1.83-1.87(\mathrm{~m}$, $\left.2 \mathrm{H}, \mathrm{CH}_{2}\right), 1.73-1.78\left(\mathrm{~m}, 2 \mathrm{H}, \mathrm{CH}_{2}\right)$.

${ }^{13} \mathrm{C}$ NMR (176 MHz, DMSO- $\left.d_{6}\right): \delta=19.5,20.0,21.0,26.6,48.9,49.4$, 104.6, 108.1, 112.7, 128.2, 129.5, 129.9, 130.3, 133.5, 133.7, 140.1, $149.0,159.8,166.1,166.5,196.5$.

HRMS: $m / z[\mathrm{M}+\mathrm{H}]^{+}$calcd for $\mathrm{C}_{21} \mathrm{H}_{19} \mathrm{NO}_{6}$ : 382.1285; found: 382.1296 .

\section{2-(8-Hydroxyjulolidine-9-carbonyl)terephthalic Acid (3b)}

Yield: $1.5 \mathrm{~g}$ (40\%); yellow foam; $R_{f}=0.25\left(\mathrm{CHCl}_{3} / \mathrm{MeOH} / \mathrm{Et}_{3} \mathrm{~N}, 70: 25: 5\right)$.
${ }^{1} \mathrm{H}$ NMR (700 MHz, DMSO- $d_{6}$ ): $\delta=12.97$ (br. s., $2 \mathrm{H}, \mathrm{COOH}$ ), 12.80 (s, $1 \mathrm{H}, \mathrm{OH}), 8.46(\mathrm{~d}, J=1.3 \mathrm{~Hz}, 1 \mathrm{H}, \mathrm{Ar}), 8.18$ (dd, $J=7.7,1.5 \mathrm{~Hz}, 1 \mathrm{H}, \mathrm{Ar}$ ), $7.47(\mathrm{~d}, J=7.9 \mathrm{~Hz}, 1 \mathrm{H}, \mathrm{Ar}), 6.38(\mathrm{~s}, 1 \mathrm{H}, \mathrm{Ar}), 3.23-3.27\left(\mathrm{~m}, 4 \mathrm{H}, \mathrm{CH}_{2}\right)$, $2.59\left(\mathrm{t}, J=6.4 \mathrm{~Hz}, 2 \mathrm{H}, \mathrm{CH}_{2}\right), 2.41\left(\mathrm{t}, J=6.0 \mathrm{~Hz}, 2 \mathrm{H}, \mathrm{CH}_{2}\right), 1.82-1.88(\mathrm{~m}$, $\left.2 \mathrm{H}, \mathrm{CH}_{2}\right), 1.72-1.79\left(\mathrm{~m}, 2 \mathrm{H}, \mathrm{CH}_{2}\right)$.

${ }^{13} \mathrm{C}$ NMR $\left(176 \mathrm{MHz}\right.$, DMSO- $\left.d_{6}\right): \delta=19.5,20.0,21.0,26.6,48.9,49.4$, $104.6,108.1,112.7,128.3,129.5,130.1,130.7,131.5,132.5,143.8$, 149.0, 159.7, 166.2, 171.9, 196.8.

HRMS: $m / z[\mathrm{M}+\mathrm{H}]^{+}$calcd for $\mathrm{C}_{21} \mathrm{H}_{19} \mathrm{NO}_{6}$ : 382.1285; found: 382.1281 .

\section{5(6)-Carboxy-X-rhodamines $4 a$ and $4 b^{6}$}

The product from the previous stage $(3 \mathbf{b} ; 1.15 \mathrm{~g}, 3 \mathrm{mmol}$ ) and 8-hydroxyjulolidine $(0.83 \mathrm{~g}, 4.4 \mathrm{mmol})$ were dissolved in DMF $(25 \mathrm{~mL})$. Trimethylsilylpolyphosphate solution in chloroform $(7 \mathrm{~mL}$ ) (see above) was added, and mixture was heated to reflux for $3 \mathrm{~h}$. The solvent was evaporated, the residue was dissolved in $\mathrm{NaOH}(5 \%, 26.5 \mathrm{~mL})$ and the mixture stirred at r.t. overnight. The solution was then diluted with $\mathrm{HCl}(36 \%, 3.6 \mathrm{~mL})$, and the solid was filtered off, washed with water $(20 \mathrm{~mL})$, and air-dried. The product was purified by flash chromatography (gradient of $\mathrm{MeOH}$ in $\mathrm{CHCl}_{3} / \mathrm{Et}_{3} \mathrm{~N}$ from 10:85:5 to 40:55:5).

\section{5-Carboxy-X-rhodamines $\left(4 \mathrm{a} \cdot \mathrm{Et}_{\mathbf{3}} \mathrm{N}\right)^{6}$}

Yield: $1.15 \mathrm{~g}$ (60\%); purple solid; m.p. $>250{ }^{\circ} \mathrm{C}$ (decomp.); $R_{f}=0.34$ $\left(\mathrm{CHCl}_{3} / \mathrm{MeOH} / \mathrm{Et}_{3} \mathrm{~N}, 70: 25: 5\right)$.

${ }^{1} \mathrm{H}$ NMR $\left(700 \mathrm{MHz}\right.$, DMSO- $\left.d_{6}\right): \delta=8.38(\mathrm{~s}, 1 \mathrm{H}, \mathrm{Ar}), 8.18(\mathrm{~d}, J=8.1 \mathrm{~Hz}$, $1 \mathrm{H}, \mathrm{Ar}), 7.17$ (d, J = 7.9 Hz, $1 \mathrm{H}, \mathrm{Ar}), 6.15(\mathrm{~s}, 2 \mathrm{H}, \mathrm{Ar}), 3.23(\mathrm{t}, J=5.4 \mathrm{~Hz}$, $4 \mathrm{H}, \mathrm{CH}_{2}$ ), 3.19 (br. s., $\left.4 \mathrm{H}, \mathrm{CH}_{2}\right), 2.86-2.89\left(\mathrm{~m}, 4 \mathrm{H}, \mathrm{CH}_{2}\right), 2.75-2.81$ (m, $\left.6 \mathrm{H}, \mathrm{CH}_{2}\right), 2.45-2.50\left(\mathrm{~m}, 4 \mathrm{H}, \mathrm{CH}_{2}\right), 1.94-1.98\left(\mathrm{~m}, 4 \mathrm{H}, \mathrm{CH}_{2}\right), 1.76-1.80$ (m, $\left.4 \mathrm{H}, \mathrm{CH}_{2}\right), 1.07\left(\mathrm{t}, \mathrm{J}=7.2 \mathrm{~Hz}, 9 \mathrm{H}, \mathrm{CH}_{3}\right)$.

\section{6-Carboxy-X-rhodamines $\left(4 \mathrm{~b} \cdot \mathrm{Et}_{3} \mathrm{~N}\right)^{6}$}

Yield: $1.4 \mathrm{~g}$ (73\%); purple solid; m.p. $>250{ }^{\circ} \mathrm{C}$ (decomp.); $R_{f}=0.16(\mathrm{CH}-$ $\left.\mathrm{Cl}_{3} / \mathrm{MeOH} / \mathrm{Et}_{3} \mathrm{~N}, 70: 25: 5\right)$.

${ }^{1} \mathrm{H}$ NMR (700 MHz, DMSO- $d_{6}$ ): $\delta=8.07$ (d, $\left.J=7.8 \mathrm{~Hz}, 1 \mathrm{H}, \mathrm{Ar}\right), 7.88$ (d, $J=7.9 \mathrm{~Hz}, 1 \mathrm{H}, \mathrm{Ar}), 7.48(\mathrm{~s}, 1 \mathrm{H}, \mathrm{Ar}), 6.12(\mathrm{~s}, 2 \mathrm{H}, \mathrm{Ar}), 3.21(\mathrm{t}, J=5.5 \mathrm{~Hz}$, $\left.4 \mathrm{H}, \mathrm{CH}_{2}\right), 3.17\left(\mathrm{t}, J=5.2 \mathrm{~Hz}, 4 \mathrm{H}, \mathrm{CH}_{2}\right), 2.88\left(\mathrm{t}, J=6.5 \mathrm{~Hz}, 4 \mathrm{H}, \mathrm{CH}_{2}\right)$, 2.68 (br. s., $\left.6 \mathrm{H}, \mathrm{CH}_{2}\right), 2.45-2.49\left(\mathrm{~m}, 4 \mathrm{H}, \mathrm{CH}_{2}\right), 1.95-1.99\left(\mathrm{~m}, 4 \mathrm{H}, \mathrm{CH}_{2}\right)$, 1.77-1.81 (m, $\left.4 \mathrm{H}, \mathrm{CH}_{2}\right), 1.02\left(\mathrm{t}, J=7.2 \mathrm{~Hz}, 9 \mathrm{H}, \mathrm{CH}_{3}\right)$.

\section{5(6)-(3-Azidopropylaminocarbonyl)-X-rhodamine $5 \mathrm{a}$ and $\mathbf{5 b}$}

To a stirred solution of the product from the previous stage $(\mathbf{4 b} ; 1 \mathrm{~g}$, $1.6 \mathrm{mmol})$ in THF (150 mL), DIC ( $2 \mathrm{mmol})$ and NHS (2 mmol) were added, and the mixture was stirred at r.t. for 5 days. The solution was evaporated and the product was purified by flash column chromatography $\left(\mathrm{CHCl}_{3} / \mathrm{MeOH}, 80: 20 ; R_{f} \mathrm{ca} .0 .25\right)$. The product was dissolved in THF ( $50 \mathrm{~mL})$, 3-azidopropylamine ( $2 \mathrm{mmol})$ was added, and the mixture was stirred at r.t. for $1 \mathrm{~h}$. Then, $\mathrm{AcOH}(0.1 \mathrm{~mL})$ was added and the mixture was stirred $30 \mathrm{~min}$. The solution was evaporated and the product was purified by column chromatography $\left(\mathrm{CHCl}_{3} / \mathrm{MeOH} / \mathrm{Et}_{3} \mathrm{~N}\right.$, 85:10:5).

\section{5-(3-Azidopropylaminocarbonyl)-X-rhodamine (5a)}

Yield: $340 \mathrm{mg}$ (35\%); purple solid; m.p. $>250{ }^{\circ} \mathrm{C}$ (decomp.); $R_{f}=0.33$ $\left(\mathrm{CHCl}_{3} / \mathrm{MeOH} / \mathrm{Et}_{3} \mathrm{~N}, 85: 10: 5\right)$.

${ }^{1} \mathrm{H}$ NMR $\left(700 \mathrm{MHz}\right.$, DMSO- $\left.d_{6}\right): \delta=8.83(\mathrm{t}, J=5.5 \mathrm{~Hz}, 1 \mathrm{H}, \mathrm{NH}), 8.43(\mathrm{~s}$, $1 \mathrm{H}, \mathrm{Ar}$ ), 8.15 (dd, $J=8.0,1.2 \mathrm{~Hz}, 1 \mathrm{H}, \mathrm{Ar}), 7.29$ (d, $J=7.9 \mathrm{~Hz}, 1 \mathrm{H}, \mathrm{Ar}$ ), $6.13(\mathrm{~s}, 1 \mathrm{H}, \mathrm{Ar}), 3.45\left(\mathrm{t}, J=6.8 \mathrm{~Hz}, 2 \mathrm{H}, \mathrm{CH}_{2}\right), 3.36-3.41\left(\mathrm{~m}, 2 \mathrm{H}, \mathrm{CH}_{2}\right)$, 
3.24 (br. s., $4 \mathrm{H}, \mathrm{CH}_{2}$ ), 3.15-3.22 (m, $\left.4 \mathrm{H}, \mathrm{CH}_{2}\right), 2.84-2.92\left(\mathrm{~m}, 4 \mathrm{H}, \mathrm{CH}_{2}\right)$, 2.43-2.50 (m, $\left.4 \mathrm{H}, \mathrm{CH}_{2}\right), 1.94-2.00\left(\mathrm{~m}, 4 \mathrm{H}, \mathrm{CH}_{2}\right), 1.85-1.77(\mathrm{~m}, 6 \mathrm{H}$, $\mathrm{CH}_{2}$ ).

${ }^{13} \mathrm{C}$ NMR $\left(176 \mathrm{MHz}\right.$, DMSO- $\left.d_{6}\right): \delta=20.2,20.3,21.0,26.7,28.3,36.7$, 48.5, 48.8, 49.3, 106.1, 106.4, 115.6, 118.3, 124.3, 124.5, 125.3, 129.5, $130.1,132.8,135.6,145.5,148.3,165.1,167.9$.

HRMS: $m / z[\mathrm{M}+\mathrm{H}]^{+}$calcd for $\mathrm{C}_{36} \mathrm{H}_{37} \mathrm{~N}_{6} \mathrm{O}_{4}$ : 617.2871; found: 617.2878 .

\section{6-(3-Azidopropylaminocarbonyl)-X-rhodamine (5b) $)^{9}$}

Yield: $390 \mathrm{mg}$ (40\%); purple solid; m.p. $>250{ }^{\circ} \mathrm{C}$ (decomp); $R_{f}=0.25$ $\left(\mathrm{CHCl}_{3} / \mathrm{MeOH} / \mathrm{Et}_{3} \mathrm{~N}, 85: 10: 5\right){ }^{9}$

${ }^{1} \mathrm{H}$ NMR $\left(800 \mathrm{MHz}\right.$, DMSO- $\left.d_{6}\right): \delta=8.68(\mathrm{t}, J=5.5 \mathrm{~Hz}, 1 \mathrm{H}, \mathrm{NH}), 8.11(\mathrm{~d}$, $J=8.8 \mathrm{~Hz}, 1 \mathrm{H}, \mathrm{Ar}), 8.02(\mathrm{~d}, J=7.6 \mathrm{~Hz}, 1 \mathrm{H}, \mathrm{Ar}), 7.60(\mathrm{~s}, 1 \mathrm{H}, \mathrm{Ar}), 6.11(\mathrm{~s}$, $2 \mathrm{H}, \mathrm{Ar}), 3.37$ (t, $\left.J=6.7 \mathrm{~Hz}, 2 \mathrm{H}, \mathrm{CH}_{2}\right), 3.26-3.29\left(\mathrm{~m}, 2 \mathrm{H}, \mathrm{CH}_{2}\right), 3.21-$ $3.24\left(\mathrm{~m}, 4 \mathrm{H}, \mathrm{CH}_{2}\right), 3.16-3.19\left(\mathrm{~m}, 4 \mathrm{H}, \mathrm{CH}_{2}\right), 2.85-2.91\left(\mathrm{~m}, 4 \mathrm{H}, \mathrm{CH}_{2}\right)$, 2.44-2.49 (m, $\left.4 \mathrm{H}, \mathrm{CH}_{2}\right), 1.94-1.99\left(\mathrm{~m}, 4 \mathrm{H}, \mathrm{CH}_{2}\right), 1.78-1.80(\mathrm{~m}, 4 \mathrm{H}$, $\mathrm{CH}_{2}$ ), 1.74 (quin, $J=6.9 \mathrm{~Hz}, 2 \mathrm{H}, \mathrm{CH}_{2}$ ).

\section{2-Chloro-3-hydroxy-4-methoxybenzaldehyde (7) ${ }^{4 \mathrm{a}, 4 \mathrm{~b}}$}

To a solution of isovanillin $6(25 \mathrm{~g}, 165 \mathrm{mmol})$ in anhydrous $\mathrm{CHCl}_{3}$ (300 mL, EtOH stabilizer must have been removed), $\mathrm{SO}_{2} \mathrm{Cl}_{2}(45 \mathrm{~mL}$, $200 \mathrm{mmol}$ ) was added dropwise and the mixture was stirred at r.t. for $24 \mathrm{~h}$. The solid was filtered off, washed with $\mathrm{CHCl}_{3}$, and air-dried to afford 7.

Yield: 27 g (90\%); white solid; m.p. $202-204{ }^{\circ} \mathrm{C}$ (Lit. ${ }^{4 a} 203-205^{\circ} \mathrm{C}$ ).

${ }^{1} \mathrm{H}$ NMR (300 MHz, DMSO- $d_{6}$ ): $\delta=10.19$ (s, $1 \mathrm{H}, \mathrm{CHO}$ ), 9.83 (br. s., $1 \mathrm{H}$, $\mathrm{OH}), 7.41(\mathrm{~d}, J=8.7 \mathrm{~Hz}, 1 \mathrm{H}, \mathrm{Ar}), 7.12(\mathrm{~d}, J=8.6 \mathrm{~Hz}, 1 \mathrm{H}, \mathrm{Ar}), 3.93(\mathrm{~s}$, $\left.3 \mathrm{H}, \mathrm{CH}_{3}\right)$.

\section{2-Chloro-4-methoxyresorcinol (8) ( $^{4 \mathrm{a}, 4 \mathrm{~b}}$}

To a suspension of 2-chloro-3-hydroxy-4-methoxybenzaldehyde 7 (4.66 g, $0.025 \mathrm{~mol})$ in $\mathrm{CH}_{2} \mathrm{Cl}_{2}(80 \mathrm{~mL}), \mathrm{SeO}_{2}(222 \mathrm{mg}, 0.002 \mathrm{~mol})$ and $35 \%$ aq $\mathrm{H}_{2} \mathrm{O}_{2}(5 \mathrm{~mL}, 0.05 \mathrm{~mol})$ were added. The mixture was stirred 48 $\mathrm{h}$ at r.t., then the organic layer was separated, washed with $10 \% \mathrm{NaH}-$ $\mathrm{SO}_{3}(20 \mathrm{~mL})$, dried over $\mathrm{Na}_{2} \mathrm{SO}_{4}$, filtered, and evaporated. The resulting solid was dissolved in methanolic $\mathrm{HCl}$ [obtained by addition of $\mathrm{AcCl}$ (2 $\mathrm{mL})$ to $\mathrm{MeOH}(40 \mathrm{~mL})$ ], then the solution was stirred for $60 \mathrm{~min}$ at r.t. and evaporated. The resulting oil was purified by flash chromatography (pure $\mathrm{CH}_{2} \mathrm{Cl}_{2}$ ) to afford 8 .

Yield: $3.9 \mathrm{~g}$ (89\%); white solid; m.p. $75-77^{\circ} \mathrm{C}$ (Lit. ${ }^{4 b} 76-77^{\circ} \mathrm{C}$ ).

${ }^{1} \mathrm{H}$ NMR (300 MHz, DMSO- $\left.d_{6}\right): \delta=9.37(\mathrm{~s}, 1 \mathrm{H}, \mathrm{OH}), 9.12(\mathrm{~s}, 1 \mathrm{H}, \mathrm{OH})$, $6.73(\mathrm{~d}, J=8.9 \mathrm{~Hz}, 1 \mathrm{H}, \mathrm{Ar}), 6.35(\mathrm{~d}, J=8.9 \mathrm{~Hz}, 1 \mathrm{H}, \mathrm{Ar}), 3.71(\mathrm{~s}, 3 \mathrm{H}$, $\mathrm{CH}_{3}$ ).

\section{4',5'-Dichloro-2',7'-dimethoxy-5(6)-carboxyfluorescein (9) ${ }^{4 a, 4 b}$}

To a solution of 2-chloro-4-methoxyresorcinol 8 (4.05 g, $23 \mathrm{mmol})$ and 4-carboxyphthalic anhydride $\mathbf{1}(2.23 \mathrm{~g}, 11.5 \mathrm{mmol})$ in methanesulfonic acid $(12 \mathrm{~mL})$ was added $\mathrm{SnCl}_{4}(1.4 \mathrm{~mL}, 12 \mathrm{mmol})$ and the mixture was stirred at $40{ }^{\circ} \mathrm{C}$ for $6 \mathrm{~h}$. After cooling to r.t., the reaction mixture was poured into ice-water $(200 \mathrm{~mL})$. The solid was filtered off, dissolved in $\mathrm{NaOH}(15 \%, 50 \mathrm{~mL})$, and the solution was acidified to $\mathrm{pH} 2$ with $\mathrm{HCl}$ (17\%). The precipitate formed was collected and airdried. The product was purified by column chromatography (gradient of $\mathrm{NH}_{3}$ in EtOH from 10:90 to 25:75). The obtained mixture of isomers was dissolved in water and the solution was acidified to $\mathrm{pH} 2$ with concentrated $\mathrm{HCl}$. The precipitate was collected and air-dried to afford a mixture $\mathbf{9 a}$ and $\mathbf{9 b}$.
Yield: $3.9 \mathrm{~g}$ (65\%); red solid.

${ }^{1} \mathrm{H}$ NMR [700 MHz, $\mathrm{D}_{2} \mathrm{O}+\mathrm{NH}_{3}$ (traces)]: $\delta=8.25(\mathrm{~s}, 1 \mathrm{H}, \mathrm{Ar}), 8.16-8.23$ (m, $2 \mathrm{H}, \mathrm{Ar}), 8.10(\mathrm{~d}, J=8.3 \mathrm{~Hz}, 1 \mathrm{H}, \mathrm{Ar}), 7.88(\mathrm{~d}, J=7.9 \mathrm{~Hz}, 1 \mathrm{H}, \mathrm{Ar})$, 7.58 (br. s., $1 \mathrm{H}, \mathrm{Ar}$ ), 6.43 (s, $2 \mathrm{H}, \mathrm{Ar}$ ), 6.27 (br. s., $2 \mathrm{H}, \mathrm{Ar}$ ), 3.65 (s, $6 \mathrm{H}$, $\mathrm{CH}_{3}$ ), 3.59 (br. s., $6 \mathrm{H}, \mathrm{CH}_{3}$ ).

\section{3',6'-Diacetoxy-4',5'-dichloro-2',7'-dimethoxy-5(6)-carboxyfluo-} rescein (10)

The product of the previous stage (mixture of $\mathbf{9 a}$ and $\mathbf{9 b})(2 \mathrm{~g}, 4$ $\mathrm{mmol})$ and pyridine $(3 \mathrm{~mL})$ in acetic anhydride $(28 \mathrm{~mL})$ was stirred for $2 \mathrm{~h}$ at $120^{\circ} \mathrm{C}$. After cooling to r.t. the solvent was evaporated, the residue was dissolved in EtOAc $(250 \mathrm{~mL})$, and the solution was washed with brine $(4 \times 50 \mathrm{~mL})$ and dried over $\mathrm{Na}_{2} \mathrm{SO}_{4}$. After filtration, the solvent was evaporated and the product obtained was used in the next stage without additional purification.

${ }^{1} \mathrm{H}$ NMR (300 MHz, DMSO- $d_{6}$ ): $\delta=8.48(\mathrm{~d}, J=0.7 \mathrm{~Hz}, 1 \mathrm{H}, \mathrm{Ar}), 8.26-$ $8.34(\mathrm{~m}, 2 \mathrm{H}, \mathrm{Ar}), 8.16-8.20(\mathrm{~m}, 1 \mathrm{H}, \mathrm{Ar}), 7.98(\mathrm{~s}, 1 \mathrm{H}, \mathrm{Ar}), 7.64(\mathrm{~d}, J=$ $8.1 \mathrm{~Hz}, 1 \mathrm{H}, \mathrm{Ar}), 6.46$ (s, $2 \mathrm{H}, \mathrm{Ar}), 6.42(\mathrm{~s}, 2 \mathrm{H}, \mathrm{Ar}), 3.56-3.61(\mathrm{~m}, 12 \mathrm{H}$, $\left.\mathrm{CH}_{3}\right), 2.38\left(\mathrm{~s}, 12 \mathrm{H}, \mathrm{CH}_{3}\right)$.

5(6)-Carboxy-3',6'-diacetoxy-4',5'-dichloro-2',7'-dimethoxyfluorescein $N$-Oxysuccinimide Ester (11a and 11b)

The product of the previous stage (mixture of 10a and 10b) was dissolved in $\mathrm{CH}_{2} \mathrm{Cl}_{2}(100 \mathrm{~mL})$. To the solution were added NHS $(0.85 \mathrm{~g}, 6.4$ $\mathrm{mmol})$ and DIC $(0.88 \mathrm{~g}, 7 \mathrm{mmol})$ and the reaction mixture was stirred for $24 \mathrm{~h}$ at r.t. The solvent was evaporated and the product was purified by flash chromatography (toluene/EtOAc, 50:50). The mixture of isomers thus obtained was separated by column chromatography (gradient of EtOAc in toluene from 20:80 to 40:60).

5-Carboxy-3',6'-diacetoxy-4',5'-dichloro-2',7'-dimethoxyfluorescein $\mathrm{N}$-Oxysuccinimide Ester (11a)

Yield: $490 \mathrm{mg}$ (18\%); purple solid; m.p. $200{ }^{\circ} \mathrm{C}$ (decomp.); $R_{f}=0.28$ (toluene/EtOAc, 1:1)

${ }^{1} \mathrm{H}$ NMR $\left(700 \mathrm{MHz}, \mathrm{CDCl}_{3}\right): \delta=8.83(\mathrm{~s}, 1 \mathrm{H}, \mathrm{Ar}$ ), 8.44 (dd, $J=8.0$, $1.4 \mathrm{~Hz}, 1 \mathrm{H}, \mathrm{Ar}), 7.42(\mathrm{~d}, J=8.1 \mathrm{~Hz}, 1 \mathrm{H}, \mathrm{Ar}), 6.19(\mathrm{~s}, 2 \mathrm{H}, \mathrm{Ar}), 3.65$ (s, $\left.6 \mathrm{H}, \mathrm{CH}_{3}\right), 2.96$ (br. s., $\left.4 \mathrm{H}, \mathrm{CH}_{2}\right), 2.40\left(\mathrm{~s}, 6 \mathrm{H}, \mathrm{CH}_{3}\right)$.

${ }^{13} \mathrm{C}$ NMR $\left(176 \mathrm{MHz}, \mathrm{CDCl}_{3}\right): \delta=20.2,25.7,56.6,106.7,115.2,118.2$, $125.3,126.1,128.0,128.2,129.0,137.2,139.6,141.4,149.1,160.3$, $167.0,167.3,168.8$

HRMS: $m / z[\mathrm{M}+\mathrm{H}]^{+}$calcd for $\mathrm{C}_{31} \mathrm{H}_{21} \mathrm{Cl}_{2} \mathrm{NO}_{13}$ : 686.0463; found: 686.0471 .

6-Carboxy-3',6'-diacetoxy-4',5'-dichloro-2',7'-dimethoxyfluorescein $\mathbf{N}$-Oxysuccinimide Ester (11b)

Yield: $990 \mathrm{mg}$ (36\%); purple solid; m.p. $195{ }^{\circ} \mathrm{C}$ (decomp.); $R_{f}=0.48$ (toluene/EtOAc, 1:1)

${ }^{1} \mathrm{H}$ NMR $\left(700 \mathrm{MHz}, \mathrm{CDCl}_{3}\right): \delta=8.41(\mathrm{dd}, J=8.0,1.2 \mathrm{~Hz}, 1 \mathrm{H}, \mathrm{Ar}), 8.20$ $(\mathrm{d}, J=8.1 \mathrm{~Hz}, 1 \mathrm{H}, \mathrm{Ar}), 7.95(\mathrm{~s}, 1 \mathrm{H}, \mathrm{Ar}), 6.20(\mathrm{~s}, 2 \mathrm{H}, \mathrm{Ar}), 3.65(\mathrm{~s}, 6 \mathrm{H}$, $\mathrm{CH}_{3}$ ), 2.90 (br. s., $4 \mathrm{H}, \mathrm{CH}_{2}$ ), 2.39 (s, $6 \mathrm{H}, \mathrm{CH}_{3}$ ).

${ }^{13} \mathrm{C}$ NMR $\left(176 \mathrm{MHz}, \mathrm{CDCl}_{3}\right): \delta=20.2,25.6,56.5,106.7,115.2,118.2$, $125.3,126.1,128.2,129.0,129.9,132.0,132.4,139.6,141.4,149.1$ $160.3,167.2,167.4,168.6$.

HRMS: $m / z[\mathrm{M}+\mathrm{H}]^{+}$calcd for $\mathrm{C}_{31} \mathrm{H}_{21} \mathrm{Cl}_{2} \mathrm{NO}_{13}$ : 686.0463; found: 686.0470 . 
5(6)-(3-Azidopropylaminocarbonyl)-4',5'-dichloro-2',7'-dimethoxyfluorescein (12a and 12b)

The product of the previous stage (11b; $280 \mathrm{mg}, 0.4 \mathrm{mmol})$ was dissolved in THF $(20 \mathrm{~mL})$, 3-azidopropylamine $(320 \mathrm{mg}, 3.2 \mathrm{mmol})$ was added, and the mixture was stirred at r.t. for $8 \mathrm{~h}$. The solution was evaporated, the residue was dissolved in $\mathrm{H}_{2} \mathrm{O}(3 \mathrm{~mL})$, and the solution was acidified to $\mathrm{pH} 2$ with concentrated $\mathrm{HCl}$ and stirred at r.t. for 30 min. The solid was filtered off, washed with water $(0.5 \mathrm{~mL})$ and airdried. The product was purified by column chromatography (gradient of $\mathrm{MeOH}$ in $\mathrm{CHCl}_{3} / \mathrm{Et}_{3} \mathrm{~N}$ from 20:75:5 to 30:65:5). The product obtained was dissolved in $\mathrm{H}_{2} \mathrm{O}(3 \mathrm{~mL})$, and the solution was acidified to $\mathrm{pH} 2$ with concentrated $\mathrm{HCl}$ and stirred at r.t. for $30 \mathrm{~min}$. The solid produced was filtered off, washed with water $(0.5 \mathrm{~mL})$ and air-dried.

\section{5-(3-Azidopropylaminocarbonyl)-4',5'-dichloro-2',7'-dimethoxy-} fluorescein (12a)

Yield: $180 \mathrm{mg}$ (75\%); purple solid; m.p. $260{ }^{\circ} \mathrm{C}$ (decomp.); $R_{f}=0.45$ $\left(\mathrm{CHCl}_{3} / \mathrm{MeOH} / \mathrm{Et}_{3} \mathrm{~N}, 65: 30: 5\right)$.

${ }^{1} \mathrm{H}$ NMR [700 MHz, $\mathrm{D}_{2} \mathrm{O}+\mathrm{NH}_{3}$ (traces)]: $\delta=8.16(\mathrm{~d}, J=1.8 \mathrm{~Hz}, 1 \mathrm{H}, \mathrm{Ar}$ ), 7.99 (dd, $J=7.9,1.8 \mathrm{~Hz}, 1 \mathrm{H}, \mathrm{Ar}), 7.33(\mathrm{~d}, J=7.9 \mathrm{~Hz}, 1 \mathrm{H}, \mathrm{Ar}), 6.22(\mathrm{~s}$, $2 \mathrm{H}, \mathrm{Ar}), 3.61\left(\mathrm{~s}, 6 \mathrm{H}, \mathrm{CH}_{3}\right), 3.56\left(\mathrm{t}, J=6.7 \mathrm{~Hz}, 2 \mathrm{H}, \mathrm{CH}_{2}\right), 3.53(\mathrm{t}, J=$ $6.6 \mathrm{~Hz}, 2 \mathrm{H}, \mathrm{CH}_{2}$ ), 1.98 (quin, $J=6.7 \mathrm{~Hz}, 2 \mathrm{H}, \mathrm{CH}_{2}$ ).

${ }^{13} \mathrm{C}$ NMR [176 MHz, $\mathrm{D}_{2} \mathrm{O}+\mathrm{NH}_{3}$ (traces)]: $\delta=27.8,37.6,49.0,55.4$, 102.7, 107.9, 110.4, 127.2, 127.7, 131.2, 134.6, 135.0, 140.6, 149.5, 151.4, 152.7, 166.9, 169.7, 173.7.

HRMS: $m / z[\mathrm{M}+\mathrm{H}]^{+}$calcd for $\mathrm{C}_{26} \mathrm{H}_{21} \mathrm{Cl}_{2} \mathrm{~N}_{4} \mathrm{O}_{8}$ : 587.0731; found: 587.0734 .

\section{6-(3-Azidopropylaminocarbonyl)-4',5'-dichloro-2',7'-dimethoxy-} fluorescein (12b)

Yield: $200 \mathrm{mg}$ (83\%); purple solid; m.p. $250{ }^{\circ} \mathrm{C}$ (decomp.); $R_{f}=0.33$ $\left(\mathrm{CHCl}_{3} / \mathrm{MeOH} / \mathrm{Et}_{3} \mathrm{~N}, 65: 30: 5\right)$.

${ }^{1} \mathrm{H}$ NMR [700 MHz, $\mathrm{D}_{2} \mathrm{O}+\mathrm{NH}_{3}$ (traces)]: $\delta=8.38(\mathrm{~d}, J=1.1 \mathrm{~Hz}, 1 \mathrm{H}, \mathrm{Ar})$, $8.02(\mathrm{dd}, J=8.2,1.6 \mathrm{~Hz}, 1 \mathrm{H}, \mathrm{Ar}), 7.89(\mathrm{~d}, J=8.1 \mathrm{~Hz}, 1 \mathrm{H}, \mathrm{Ar}), 6.30$ (s, $2 \mathrm{H}, \mathrm{Ar}), 3.61\left(\mathrm{~s}, 6 \mathrm{H}, \mathrm{CH}_{3}\right), 3.58\left(\mathrm{t}, J=6.6 \mathrm{~Hz}, 2 \mathrm{H}, \mathrm{CH}_{2}\right), 3.54(\mathrm{t}, J=$ $6.7 \mathrm{~Hz}, 2 \mathrm{H}, \mathrm{CH}_{2}$ ), 1.99 (quin, $J=6.6 \mathrm{~Hz}, 2 \mathrm{H}, \mathrm{CH}_{2}$ ).

${ }^{13} \mathrm{C}$ NMR [176 MHz, $\mathrm{D}_{2} \mathrm{O}+\mathrm{NH}_{3}$ (traces)]: $\delta=27.9,37.6,49.0,55.4$, 103.2, 107.6, 110.8, 128.2, 128.7, 130.0, 131.7, 134.7, 142.9, 149.5, 151.2, 152.8, 166.7, 169.8, 174.0.

HRMS: $m / z[\mathrm{M}+\mathrm{H}]^{+}$calcd for $\mathrm{C}_{26} \mathrm{H}_{21} \mathrm{Cl}_{2} \mathrm{~N}_{4} \mathrm{O}_{8}$ : 587.0731; found: 587.0744 .

\section{Funding Information}

The authors gratefully acknowledge support from the Russian Foundation for Basic Research, grant 18-03-00094-a. Experiments were in part carried out using the equipment provided by the IBCH core facility (CKP IBCH, supported by Russian Ministry of Education and Science, grant RFMEFI62117X0018).

\section{Supporting Information}

Supporting information for this article is available online at https://doi.org/10.1055/s-0037-1610216.

\section{References}

(1) (a) Christie, R. M. Rev. Prog. Color. 1993, 23, 1. (b) Schwander, H.; Hebdrix, C. Ullmann's Encyclopedia of Industrial Chemistry 2000, 15, 367. (c) Beija, M.; Afonso, C. A. M.; Martinho, J. M. G. Chem. Soc. Rev. 2009, 38, 2410. (d) Terai, T.; Nagano, T. Eur. J. Physiol. 2013, 46, 347.

(2) (a) Ueno, Y.; Jiao, G.-S.; Burgess, K. Synthesis 2004, 2591. (b) Brunet, A.; Aslam, T.; Bradley, M. Bioorg. Med. Chem. Lett. 2014, $24,3186$.

(3) (a) Kvach, M. V.; Stepanova, I. A.; Prokhorenko, I. A.; Stupak, A. P.; Bolibrukh, D. A.; Korshun, V. A.; Shmanai, V. V. Bioconjugate Chem. 2009, 20, 1673. (b) Fu, M.; Zhang, X.; Wang, J.; Chen, H.; Gao, Y. Curr. Org. Chem. 2016, 20, 1584.

(4) (a) Lyttle, M. H.; Carter, T. G.; Cook, R. M. Org. Process Res. Dev. 2001, 5, 45. (b) Tsybulsky, D. A.; Kvach, M. V.; Stepanova, I. A.; Korshun, V. A.; Shmanai, V. V. J. Org. Chem. 2012, 77, 977. (c) Nåbo, L. J.; Madsen, C. S.; Jensen, K. J.; Kongsted, J.; Astakhova, K. ChemBioChem 2015, 16, 1163.

(5) (a) Dwight, S. J.; Levin, S. Org. Lett. 2016, 18, 5316. (b) Dwight, S. J.; Levin, S. PCT Int. Appl. WO 2017/059308, 2017. (c) Gemma Mudd, G.; Pérez, I.; Fethers, N.; Dodd, P. G.; Barbeau, O.; Auer, M. Methods Appl. Fluoresc. 2015, 4, 045002.

(6) Uddin, M. J.; Marnett, L. J. Org. Lett. 2008, 10, 4799.

(7) (a) Sun, W.-C.; Gee, K. R.; Klaubert, D. H.; Haugland, R. P. J. Org. Chem. 1997, 62, 6469. (b) Wu, X.; Tian, M.; Fan, W.; Pan, Y.; Zhai, Y.; Niu, Y.; Li, C.; Lu, T.; Mei, Q. J. Fluoresc. 2014, 24, 775. (c) Tian, M.; Wu, X.; Zhang, B.; Li, J.; Shi, Z. Bioorg. Med. Chem. Lett. 2008, $18,1977$.

(8) (a) Liboska, R.; Ligasova, A.; Strunin, D.; Rosenberg, I.; Koberna, K. PLoS ONE 2012, e51679. (b) Astakhova, K.; Wengel, J. Chem. Eur. J. 2012, 19, 1112. (c) Faulds, K.; Smith, E.; Graham, D. Anal. Chem. 2004, 76, 412.

(9) Farzan, V. M.; Ulashchik, E. A.; Martynenko-Makaev, Y. V.; Kvach, M. V.; Aparin, I. O.; Brylev, V. A.; Prikazchikova, T. A.; Maklakova, S. Y.; Majouga, A. G.; Ustinov, A. V.; Shipulin, G. A.; Shmanai, V. V.; Korshun, V. A.; Zatsepin, T. S. Bioconjugate Chem. 2017, 28, 2599.

(10) (a) Zhang, S.; Fan, J.; Zhang, S.; Wang, J.; Wang, X.; Peng, X. Chem. Commun. 2014, 14021. (b) Debnath, T.; Maity, P.; Lobo, H.; Singh, B.; Shankarling, G. S.; Ghosh, H. N. Chem. Eur. J. 2014, $20,3510$.

(11) Butkevich, A. N.; Belov, V. N.; Sokolov, V. V.; Shojaei, H.; Sidenstein, S. C.; Kamin, D.; Matthias, J.; Vlijm, R.; Engelhardt, J.; Hell, S. W. Chem. Eur. J. 2017, 23, 12114. 